\title{
Compatibility effects based on stimulus and response numerosity
}

\author{
JEFF MILLER \\ University of Otago, Dunedin, New Zealand \\ STEPHEN G. ATKINS \\ Massey University, Auckland, New Zealand \\ and \\ FENNA VAN NES \\ University of Leiden, Leiden, The Netherlands
}

\begin{abstract}
Four choice reaction time experiments documented a stimulus-response (S-R) compatibility effect involving the numbers of stimuli and responses. In Experiment 1, the stimulus consisted of one or two tones, and the correct response was either one or two taps of a response key. Responses were much faster with a compatible S-R assignment, in which the number of taps matched the number of tones, than with an incompatible assignment in which these numbers mismatched. Experiments 2 and 3 replicated this effect, using visual stimuli and bimodal stimuli, respectively, suggesting that auditory/manual rhythmic compatibility is not essential to it. Experiment 4 showed that an analogous but smaller effect is obtained when stimuli are the digits 1 and 2 . This new numerosity-based compatibility effect has general theoretical implications regarding the mechanisms responsible for compatibility effects and practical implications for interface design.
\end{abstract}

Some of the strongest effects in choice reaction time (RT) research arise from manipulations of the assignments of stimuli to responses, and such effects are generally referred to as stimulus-response (S-R) compatibility effects (e.g., Welford, 1980). The modern study of compatibility effects was stimulated mainly by the pioneering studies of P. M. Fitts and his colleagues (e.g., Fitts \& Seeger, 1953).

Studies of S-R compatibility have generally focused on effects involving spatial location or verbal coding. For example, if the stimuli are lights to the left or the right of fixation and the responses are keypresses with the left or the right hand, people respond much more rapidly and accurately if they are instructed to respond to each light with the hand on the same side (compatible condition) than if they are instructed to respond with the hand on the opposite side (incompatible condition; see, e.g., Shulman $\&$ McConkie, 1973). Similarly, spoken responses to name-

This research was supported by a Marsden grant from the Royal Society of New Zealand to J.M. The authors thank Bernie Caessens, Arthur Glenberg, Sylvan Kornblum, Jeffrey Toth, and an anonymous reviewer for constructive comments on earlier versions of the article. Correspondence concerning this article should be addressed to J. Miller, Department of Psychology, University of Otago, Dunedin, New Zealand (e-mail: miller@psy.otago.ac.nz). able stimuli are faster if the response matches the stimulus name than if it does not (e.g., Sanders, 1970).

S-R compatibility effects have been studied extensively because they are of both practical and theoretical interest. Practically, information about the performance consequences of alternative $\mathrm{S}-\mathrm{R}$ assignments has implications for the design of man-machine interfaces (see, e.g., Proctor \& Reeve, 1990). Reviews of RT research within the literature on human factors often list sources of S-R compatibility effects that should be taken into account in interface design (e.g., Wickens, 1987). Theoretically, interest has centered on the mechanisms responsible for the effects of compatibility (e.g., Kornblum, Hasbroucq, \& Osman, 1990). Several explanations of compatibility effects have been proposed, many relying on the notion that these effects arise primarily during the translation of a stimulus representation to its associated response. According to this view, compatibility effects arise because this translation occurs more rapidly when the stimulus and the response share certain common physical or conceptual characteristics than when they do not (e.g., Kornblum et al., 1990; Reeve \& Proctor, 1990; Teichner \& Krebs, 1974).

This article reports four experiments providing evidence of a strong new S-R compatibility effect involving stimulus and response numerosity. There has been considerable interest in number processing, especially the 
perception of numerosity (e.g., Viviani, 1979), the performance of numerical operations (e.g., Zbrodoff \& Logan, 1990), and the biological underpinnings of number representation (e.g., Dehaene \& Cohen, 1997). There have been no studies in which both the perception of numerosity and the production of specified numbers of responses have been considered, so the potential S-R compatibility effects along this dimension demonstrated in this study have not previously been noted.

\section{EXPERIMENT 1}

In each trial, the participants were presented with one or two tones and were required to respond by tapping a key once or twice. In the compatible condition, the number of taps matched the number of tones, whereas in the incompatible condition these numbers mismatched.

\section{Method}

Participants. The participants were 8 volunteers ( 3 females, 7 right-handed) recruited at the University of Otago. They ranged in age from 20 to 42 years $(M=24.8$ years $)$. Each participant was paid NZ \$6 to participate in a single session lasting approximately $35 \mathrm{~min}$.

Apparatus and Stimuli. The stimuli were presented and the responses and RTs were recorded by an IBM-compatible computer. The stimuli were $800-\mathrm{Hz}$ tones presented binaurally over headphones for $40 \mathrm{msec}$ at approximately $50 \mathrm{~dB}$. When two tones were presented, they were separated by a gap of $20 \mathrm{msec}$. Responses were made by tapping the slash (/) key on a standard computer keyboard either once or twice.

Procedure. Each trial began with the appearance on the computer screen of a small centered rectangle, approximately $0.6^{\circ}$ high and $0.5^{\circ}$ wide, to serve as a warning signal and fixation point. The tone or tones were presented $1 \mathrm{sec}$ after the onset of this rectangle. Then the participant's keypress response(s) was recorded. RT was measured as the time from the onset of the first (or only) tone until the first (or only) tap. ${ }^{1}$ When a two-tap response was given, the intertap interval (ITI) was measured as the time from the first tap to the second tap. After the first keypress response, the computer was programmed to wait a maximum of $750 \mathrm{msec}$ for a second tap to be emitted; if no second keypress was detected during this time, the response was scored as a single tap.

Each participant was tested in eight blocks of 40 randomly ordered trials. In each block, one tone was presented in half of the trials, and two tones were presented in the other half.

Each participant was tested in four consecutive blocks with each $\mathrm{S}-\mathrm{R}$ assignment (i.e., compatible vs. incompatible), with the order of these assignments counterbalanced across participants. At the beginning of each block, instructions on the computer screen indicated the S-R mapping for that block and reminded the participants that they should respond as quickly and accurately as possible. The participants were also instructed that when they produced a two-tap response, they should make the two taps as rapidly as possible.

If a participant responded incorrectly, error feedback was given to indicate that the number of taps had been incorrect. In addition, if a response was made with an RT less than $100 \mathrm{msec}$, feedback was given telling the participant not to respond before the tone was presented.

Except as noted otherwise, these methods were also used in subsequent experiments.

\section{Results and Discussion}

Trials with RTs less than $150 \mathrm{msec}$ or greater than $2 \mathrm{sec}$ were excluded as fast and slow outliers, eliminat-
Table 1

Percentages of Correct Responses (PCs), Mean Reaction Times (RTs), and Mean Intertap Intervals (ITIs) as a Function of Compatibility, Stimuli, and Responses in Experiment 1

\begin{tabular}{cccccccc}
\hline & \multicolumn{3}{c}{ Compatible } & & \multicolumn{3}{c}{ Incompatible } \\
\cline { 2 - 4 } \cline { 6 - 7 } \cline { 5 - 6 } & S1 R1 & S2 R2 & $M$ & & S1 R2 & S2 R1 & $M$ \\
\hline PC & 98.4 & 98.4 & 98.4 & & 98.1 & 94.5 & 96.3 \\
RT & 381 & 380 & 380 & & 533 & 544 & 538 \\
ITI & & 176 & & & 199 & & \\
\hline
\end{tabular}

Note-S1 and S2 denote conditions with one and two stimulus tones, respectively. R1 and R2 denote one-tap and two-tap responses, respectively.

ing approximately $0.2 \%$ and $0.1 \%$ of the trials, respectively. Table 1 shows the mean percentages of correct responses (PCs) and the mean RTs and ITIs for trials with correct responses.

As is shown in Table 1, responses were faster and more accurate with the compatible $\mathrm{S}-\mathrm{R}$ mapping than with the incompatible one. To evaluate the statistical reliability of these effects, parallel analyses of variance (ANOVAs) were computed, using PCs and RTs. These ANOVAs had a between-subjects factor of order (compatible blocks followed by incompatible blocks vs. the reverse) and within-subjects factors of compatibility, block, and number of tones. In the analysis of RT, the effect of compatibility was highly significant $[F(1,6)=42.87$, $p<.005]$. There was also an effect of block $[F(3,18)=$ $7.11, p<.005]$, caused almost exclusively by slower responses in the first block within each compatibility condition, and no other sources of variance approached significance. The ANOVA on PCs also yielded a significant effect of compatibility $[F(1,6)=13.42, p<.02]$.

A further ANOVA was conducted on the ITIs obtained from the trials in which the two-tap response was made. Compatibility was the only significant effect in this analysis $[F(1,6)=11.55, p<.02]$. As is shown in Table 1 , the mean ITI was smaller in the compatible condition than in the incompatible one, suggesting that numerosity-based compatibility may influence response production, as well as response initiation. This effect may, however, be produced by occasional trials in which participants start to generate a single tap and add the second tap after subsequently realizing that it was required. For example, the compatibility effect on ITI merely approached significance $(.09<p<.10)$ if trials with ITIs greater than $400 \mathrm{msec}$ were removed from the analysis. ${ }^{2}$ On average, across participants and conditions, there was also a significant negative correlation between RT and ITI for correlations computed across trials within a given condition for each participant separately $(r=-.18, p<.05)$. These correlations were not modulated by compatibility. These negative correlations probably have little theoretical significance, especially given that the correlations were even weaker in the subsequent experiments (Experiment 1B, $r=-.04, p>.2$; Experiment 2, $r=-.06, p>.2$; Experiment $3, r=-.08, p<.05$ ).

In summary, these results demonstrate a numerositybased S-R compatibility effect, with faster responses 
when the numbers of stimuli and responses match than when they mismatch. ${ }^{3}$ The subsequent experiments were designed to examine the nature and generality of this phenomenon.

\section{EXPERIMENT 2}

The compatibility effect observed in Experiment 1 could potentially have been mediated by rhythmicity, rather than by numerosity. One- and two-tone stimuli clearly have different rhythmic properties, as do one- and two-tap responses. Because the rhythmic properties of the stimuli and the responses matched better in the compatible condition, RTs could have been influenced by a tendency to copy these rhythmic properties, rather than by the match versus mismatch of numerosity per se. Frontal patients tend to copy the movements of an experimenter even when instructed to produce different movements (Luria, 1966, p. 257), suggesting a strong tendency for the motor system to copy its inputs.

To test this alternative explanation, visual stimuli were used in this experiment. The stimuli were one or two response-terminated rectangles presented above or below fixation, or both simultaneously, and the participants were instructed to respond according to the number of rectangles. Simultaneous presentation in two-stimulus trials should eliminate the rhythmic properties of the stimuli and, thus, substantially reduce or eliminate any compatibility effect mediated by these rhythmic properties. The rhythmic properties of visual stimuli are also known to be less powerful than those of auditory stimuli (e.g., Glenberg \& Jona, 1991).

\section{Method}

The participants were 8 new right-handed volunteers ( 4 females), $18-43$ years of age $(M=26.8$ years $)$. The stimuli were bright rectangles $\left(2^{\circ}\right.$ high and $1.5^{\circ}$ wide) presented $1.5^{\circ}$ above and below the fixation rectangle (edge to edge), and they remained on the screen until the response was made. Each single rectangle was presented in 10 trials per block, and both were presented in 20 trials. In the compatible condition, the participants were instructed to emit one keypress if one rectangle appeared and two keypresses if two rectangles appeared; in the incompatible condition, these assignments were reversed.

\section{Results and Discussion}

Approximately $0.2 \%$ and $0.04 \%$ of the trials were eliminated as fast and slow outliers, respectively. Table 2

\section{Table 2}

Percentages of Correct Responses (PCs), Mean Reaction Times (RTs), and Mean Intertap Intervals (ITIs) as a Function of Compatibility, Stimuli, and Responses in Experiment 2

\begin{tabular}{lccccccccc}
\hline & \multicolumn{4}{c}{ Compatible } & & \multicolumn{4}{c}{ Incompatible } \\
\cline { 2 - 4 } \cline { 7 - 9 } & SU R1 & SL R1 & S2 R2 & $M$ & & SU R2 & SL R2 & S2 R1 & $M$ \\
\hline PC & 99.7 & 99.1 & 99.8 & 99.5 & & 99.4 & 99.7 & 94.7 & 97.9 \\
RT & 455 & 430 & 404 & 429 & & 542 & 523 & 507 & 524 \\
ITI & & & 177 & & & 182 & 192 & & \\
\hline
\end{tabular}

Note-SU, SL, and S2 denote the upper rectangle, the lower rectangle, and both rectangles, respectively. R1 and R2 denote one-tap and twotap responses, respectively. shows the mean PCs, RTs, and ITIs, computed as in Experiment 1 .

The means again indicate a substantial advantage for the compatible mapping over the incompatible one with respect to RT and a smaller advantage for PC. Separate ANOVAs were again conducted on RT and PC, and the stimulus factor had three levels: upper rectangle, lower rectangle, or both. The analysis of RT yielded significant effects of compatibility $[F(1,6)=31.95, p<.002]$ and stimulus $[F(2,12)=14.07, p<.002]$. Pairwise comparisons indicated that responses were faster to two rectangles than to one but that the upper and the lower rectangles were not significantly different. The ANOVA on PCs yielded a marginally significant effect of compatibility $[F(1,6)=5.87, .06>p>.05]$ but a highly significant interaction of stimulus and compatibility $[F(2,12)=$ $7.80, p<.01]$. As is evident in Table 2, there was a large effect of compatibility on accuracy only when two stimuli were presented.

A further ANOVA was conducted on the ITIs obtained from the trials in which the two-tap response was made. The 10-msec effect of compatibility did not approach significance $(p>.3)$.

\section{EXPERIMENT 3}

Both of the compatibility effects in the first two experiments were obtained in tasks with one versus two identical stimuli. Assessment of numerosity is more rapid with identical stimuli than with nonidentical ones (Frick, 1987), so numerosity-based compatibility effects might not be found in a task in which the two-stimulus displays contained two dissimilar stimuli. This experiment thus used two-stimulus displays with stimuli in different modalities in order to maximize dissimilarity. The one-stimulus trials included either an auditory stimulus or a visual stimulus, and the two-stimulus trials included both.

\section{Method}

The participants were 8 new right-handed volunteers ( 5 females) 20-34 years of age ( $M=23.8$ years). The auditory and visual stimuli were the same tone and rectangle as those used in Experiments 1 and 2, although the rectangle was always presented at fixation. Each single stimulus was presented in 10 trials per block, and both were presented simultaneously in 20 trials. In the compatible condition, the participants were instructed to emit one keypress if one stimulus was presented and two keypresses if both were presented; in the incompatible condition, these assignments were reversed.

\section{Results and Discussion}

Approximately $0.7 \%$ and $0.2 \%$ of the trials were eliminated as fast and slow outliers, respectively. Table 3 shows the averages. Responses were slower in this experiment than in the previous ones, consistent with the idea that evaluation of numerosity is slower with nonidentical stimuli (Frick, 1987), but this trend was not significant $(p>.1)$.

ANOVAs analogous to those used in Experiment 2 were conducted on the PCs and RTs, using the three stimulus conditions of auditory alone, visual alone, or 
Table 3

Percentages of Correct Responses (PCs), Mean Reaction Times (RTs), and Mean Intertap Intervals (ITIs) as a Function of Compatibility, Stimuli, and Responses in Experiment 3

\begin{tabular}{lccccccccc}
\hline & \multicolumn{4}{c}{ Compatible } & & \multicolumn{4}{c}{ Incompatible } \\
\cline { 2 - 4 } \cline { 7 - 9 } & SA R1 & SV R1 & S2 R2 & $M$ & & SA R2 & SV R2 & S2 R1 & $M$ \\
\hline PC & 97.8 & 97.8 & 97.5 & 97.7 & & 94.7 & 94.1 & 94.2 & 94.3 \\
RT & 448 & 481 & 384 & 438 & & 595 & 652 & 555 & 601 \\
ITI & & & 217 & & 231 & 235 & & \\
\hline
\end{tabular}

Note-SA, SV, and S2 denote the auditory stimulus, the visual stimulus, and both stimuli, respectively. R1 and R2 denote one-tap and twotap responses, respectively.

both. The effect of compatibility on RT was significant $[F(1,6)=9.21, p<.025]$, with responses again faster when the numbers of stimuli and responses matched than when they did not. Thus, the numerosity-based compatibility effect extends to tasks in which the two-stimulus condition includes two very dissimilar stimuli.

The effect of stimulus was also significant in the analysis of RT $[F(2,12)=8.30, p<.01]$. On average across tasks, responses were fastest to the double stimulus $(M=470 \mathrm{msec})$, intermediate to the auditory stimulus $(M=522 \mathrm{msec})$, and slowest to the visual stimulus $(M=$ $566 \mathrm{msec}$ ). Pairwise comparisons using the NewmanKeuls procedure indicated that only the fastest and slowest conditions differed reliably.

In the parallel analysis of PC, only the effect of compatibility was significant $[F(1,6)=15.18, p<.01]$. In the analysis of ITI in trials with two-tap responses, the effect of compatibility was not significant $[F(1,6)=$ $2.51, .20>p>.15]$.

\section{EXPERIMENT 4}

Like other compatibility effects, the numerosity compatibility effect may be driven in part by a match between semantic representations of 1 and 2 used in coding the stimuli and responses. To assess this purely conceptual contribution to the effect, we used the digits 1 and 2 as stimuli, assigning them compatibly or incompatibly to one- and two-tap responses.

\section{Method}

The participants were 8 new volunteers ( 6 females, 6 right-handed), $18-45$ years of age $(M=24$ years). The stimuli were the digits 1 and $2\left(0.4^{\circ}\right.$ high and $0.3^{\circ}$ wide $)$. They were presented at fixation and remained on the screen until the response was made. Each was presented in 20 trials per block. In the compatible condition, the participants were instructed to emit one keypress if 1 appeared and two keypresses if 2 appeared; in the incompatible condition, these assignments were reversed.

\section{Results and Discussion}

Table 4 shows the means, computed after eliminating approximately $0.4 \%$ and $0.1 \%$ of the trials as fast and slow outliers, respectively.

The ANOVA on RT yielded a significant effect of compatibility $[F(1,6)=66.02, p<.001]$ and a signifi- cant interaction of digit and compatibility $[F(1,6)=$ $15.32, p<.01]$, reflecting a smaller effect of compatibility for 1 than for 2 . The ANOVA on PCs revealed that responses were more accurate to 1 than to $2[F(1,6)=$ $9.00, p<.025]$ and that this advantage was larger in the incompatible condition $[F(1,6)=6.00, p<.05]$. The 18 -msec effect of compatibility on ITIs was marginally significant $[F(1,6)=4.05, p<.1]$.

\section{GENERAL DISCUSSION}

Four experiments demonstrated a new compatibility effect involving stimulus and response numerosity. Whether stimuli were auditory, visual, or bimodal, participants responded more quickly when one stimulus was assigned to a one-tap response and two stimuli were assigned to a twotap response than when these assignments were reversed. Moreover, the effect was quite large-approximately $100 \mathrm{msec}$ with visual stimuli and $150 \mathrm{msec}$ with auditory or bimodal stimuli. Although it is difficult to compare the sizes of compatibility effects across stimulus dimensions, it appears that this numerosity-based effect is at least as large as spatial compatibility effects. Shulman and McConkie (1973), for example, found a spatial compatibility effect of only approximately $50 \mathrm{msec}$ with left versus right stimulus lights and left- versus right-hand responses. In at least some cases, the numerosity compatibility effect is too large to be explained fully by conceptual coding of the stimuli and responses; for example, between-experiment comparisons indicated that the effect was reliably larger in Experiments 1 and 1B than in Experiment $4(p<.01)$.

The existence of this new compatibility effect has several implications for theories of the mechanisms underlying compatibility effects. First, some theories are, in principle, quite general across stimulus and response dimensions, specifying that compatibility effects should arise whenever the stimulus and the response are related along any dimension (e.g., Kornblum et al., 1990; Reeve \& Proctor, 1990). Clearly, the demonstration of a compatibility effect along a new dimension tends to support these theories over alternative theories that explain compatibility effects in terms of coding processes specific to a particular dimension (e.g., space; Simon, 1968). Second, compatibility effects - especially of the spatial variety-

Table 4

Percentages of Correct Responses (PCs), Mean Reaction Times (RTs), and Mean Intertap Intervals (ITIs) as a Function of Compatibility, Stimuli, and Responses in Experiment 4

\begin{tabular}{cccccccc}
\hline & \multicolumn{3}{c}{ Compatible } & & \multicolumn{3}{c}{ Incompatible } \\
\cline { 2 - 4 } \cline { 6 - 7 } \cline { 5 - 6 } & S1 R1 & S2 R2 & $M$ & & S1 R2 & S2 R1 & $M$ \\
\hline PC & 99.4 & 99.4 & 99.4 & & 99.2 & 97.3 & 98.3 \\
RT & 387 & 376 & 382 & & 447 & 474 & 460 \\
ITI & & 181 & & 199 & & \\
\hline
\end{tabular}

Note- $\mathrm{S} 1$ and S2 denote the stimulus digits 1 and 2, respectively. R1 and R2 denote one-tap and two-tap responses, respectively. 
have sometimes been attributed to processes of spatial attention (e.g., Simon, 1968). It is difficult to interpret the present numerosity-based compatibility effect in terms of such processes, however, because neither the stimulus alternatives nor the response alternatives vary with respect to the spatial focus of attention. Third, effects of spatial compatibility have sometimes been partly attributed to direct motor-level priming of the hand associated with the compatible response (cf. Eimer, 1995). Such priming cannot explain the present effects, however, because the same effector was used to make all responses. Fourth, spatial compatibility effects have sometimes been interpreted as a measure of the extent to which certain actions are afforded by particular stimuli (e.g., Michaels, 1988). Although one might similarly argue that temporal patterns such as those used in Experiment 1 give rise to a compatibility effect because they afford a mimicking response, it is difficult to extend this interpretation to Experiments 2 and 3 without making the essentially circular claim that any two stimuli afford a two-tap response.

More generally, numerosity-based compatibility effects fit well with recent theories emphasizing the commonalities of the representations used for perception and action (e.g., Hommel, Müsseler, Aschersleben, \& Prinz, 2001) and for language and action (e.g., Glenberg \& Kaschak, 2002). The present results clearly support the idea that perceptual and motor systems use common representations of numerosity, just as space-based compatibility effects indicate that they use common representations of space. This conclusion is also consistent with the idea that certain rather specific brain areas are devoted to number processing (e.g., Dehaene \& Cohen, 1997). It would be efficient for the same areas to be used for both perception and production of numerosity, and use of a common system subserving both perception and production would, of course, produce numerosity-based compatibility effects.

Another interesting aspect of the present compatibility manipulation is that it may sometimes influence motor processes, as suggested by the ITIs, although further evidence is needed to clarify these effects. Small effects of spatial compatibility on motor processes have been observed in some experiments (e.g., Simon, 1968), but not in others (e.g., Frowein \& Sanders, 1978). Motor-level effects of compatibility challenge models in which compatibility effects are localized solely within decision processes (e.g., Teichner \& Krebs, 1974), suggesting instead more dynamic models in which such effects could also propagate to subsequent motor processes (e.g., Zhang, Zhang, \& Kornblum, 1999).

Finally, the large compatibility effects obtained in these experiments identify $\mathrm{S}-\mathrm{R}$ numerosity as a dimension that must be considered in the design of man-machine interfaces. Indeed, numerosity-based compatibility is already embodied in some highly efficient interfaces. For example, musical scores generally involve a strict oneto-one correspondence between printed notes and the musician's action of sounding a note. Conversely, violation of numerosity-based compatibility may be partly re- sponsible for the limited impact of theoretically promising chord-based keyboards (e.g., Gopher \& Raij, 1988), because these keyboards require multiple keypress responses to select single characters.

\section{REFERENCES}

Dehaene, S., \& Cohen, L. (1997). Cerebral pathways for calculation: Double dissociation between rote verbal and quantitative knowledge of arithmetic. Cortex, 33, 219-250.

EIMER, M. (1995). Stimulus-response compatibility and automatic response activation: Evidence from psychophysiological studies. Journal of Experimental Psychology: Human Perception \& Performance, 21, 837-854.

FitTs, P. M., \& SeEger, C. M. (1953). S-R compatibility: Spatial characteristics of stimulus and response codes. Journal of Experimental Psychology, 46, 199-210.

FrICK, R. W. (1987). The homogeneity effect in counting. Perception \& Psychophysics, 41, 8-16.

FroweIn, H. W., \& SANDERs, A. F. (1978). Effects of visual stimulus degradation, S-R compatibility, and foreperiod duration on choice reaction time and movement time. Bulletin of the Psychonomic Society, 12, 106-108.

Glenberg, A. M., \& Jona, M. (1991). Temporal coding in rhythm tasks revealed by modality effects. Memory \& Cognition, 19, 514522.

Glenberg, A. M., \& KaschaK, M. P. (2002). Grounding language in action. Psychonomic Bulletin \& Review, 9, 558-565.

Gopher, D., \& RAIJ, D. (1988). Typing with a two-hand chord keyboard: Will the QWERTY become obsolete? IEEE Transactions on Systems, Man, \& Cybernetics, 18, 601-609.

GrosJEAN, M., \& MordKOFF, J. T. (2001). Temporal stimulus-response compatibility. Journal of Experimental Psychology: Human Perception \& Performance, 27, 870-878.

Hommel, B., Müsseler, J., Aschersleben, G., \& Prinz, W. (2001). The theory of event coding (TEC): A framework for perception and action planning. Behavioral \& Brain Sciences, 24, 849-937.

Kornblum, S., HasbroucQ, T., \& Osman, A. M. (1990). Dimensional overlap: Cognitive basis for stimulus-response compatibility-A model and taxonomy. Psychological Review, 97, 253-270.

LurIA, A. R. (1966). Higher cortical functions in man. New York: Basic Books.

Michaels, C. F. (1988). S-R compatibility between response position and destination of apparent motion: Evidence of the detection of affordances. Journal of Experimental Psychology: Human Perception \& Performance, 14, 231-240.

Proctor, R. W., \& ReEve, T. G. (1990). Stimulus-response compatibility: An integrated perspective. Amsterdam: North-Holland.

Reeve, T. G., \& Proctor, R. W. (1990). The salient features coding principle for spatial- and symbolic-compatibility effects. In R. W. Proctor \& T. G. Reeve (Eds.), Stimulus-response compatibility: An integrated perspective (pp. 163-180). Amsterdam: North-Holland.

SANDERS, A. F. (1970). Some variables affecting the relation between relative stimulus frequency and choice reaction time. Acta Psychologica, 33, 45-55.

Shulman, H. G., \& McConkie, A. (1973). S-R compatibility, response discriminability, and response codes in choice reaction time. Journal of Experimental Psychology, 98, 375-378.

SimON, J. R. (1968). Effect of ear stimulated on reaction time and movement time. Journal of Experimental Psychology, 78, 344-346.

Teichner, W. H., \& KreBs, M. J. (1974). Laws of visual choice reaction time. Psychological Review, 81, 75-98.

Viviani, P. (1979). Choice reaction time for temporal numerosity. Journal of Experimental Psychology: Human Perception \& Performance, 5, 157-167.

WELFORD, A. T. (1980). Choice reaction time: Basic concepts. In A. T. Welford (Ed.), Reaction times (pp. 73-128). New York: Academic Press.

WiCKENS, C. D. (1987). Information processing, decision-making, and cognition. In G. Salvendy (Ed.), Handbook of human factors (pp. 72107). New York: Wiley. 
Zbrodoff, N. J., \& Logan, G. D. (1990). On the relation between production and verification tasks in the psychology of simple arithmetic. Journal of Experimental Psychology: Learning, Memory, \& Cognition, 16, 83-97.

ZHANG, H., ZHANG, J., \& KoRnBlum, S. (1999). A parallel distributed processing model of stimulus-stimulus and stimulus-response compatibility. Cognitive Psychology, 38, 386-432.

\section{NOTES}

1. An exact definition of RT is actually somewhat problematic in this situation because, on single-tone trials, observers necessarily have to wait for some time before concluding that the second tone is not going to be presented. Such waiting could contribute to any observed difference in RTs to one versus two tones, thereby complicating its interpretation. Fortunately, this problem does not complicate the interpretation of the compatibility effect, because this effect is measured on the average across one- and two-tone stimuli. In addition, Experiments 2 and 3 avoided the problem by presenting stimuli simultaneously in the twostimulus conditions.
2. We are indebted to Jeff Toth for raising this issue.

3. Bernie Caessens suggested that the compatibility effect might have been driven by duration, rather than by numerosity (cf. Grosjean \& Mordkoff, 2001), because duration and numerosity were confounded (i.e., two-stimulus displays lasted longer than one-stimulus displays, as did two-keypress responses). We therefore carried out an additional Experiment $1 \mathrm{~B}$ that was exactly parallel to Experiment 1, except that the duration of the single tone was increased to $100 \mathrm{msec}$ to equate the durations of the one- and two-tone displays. Experiment $1 \mathrm{~B}$ yielded significant effects of compatibility on RT of $159 \mathrm{msec}(p<.01)$ and on ITI of $40 \mathrm{msec}(p<.05)$, with the ITI effect remaining significant even after trials with large ITIs were removed. Overall ANOVAs on PC, RT, and ITI including the results from both experiments yielded no significant effects or interactions involving the factor of experiment (i.e., 1 vs. 1B). This pattern indicates that stimulus duration is not responsible for the numerosity-based compatibility effect, as do the results of Experiments 2 and 3.

(Manuscript received April 23, 2004; revision accepted for publication July 9, 2004.) 